Original research article

\title{
Circulating levels of selected adipokines in women with gestational diabetes and type 2 diabetes
}

\author{
David Karasek ${ }^{1 *}$, Veronika Kubickova ${ }^{2}$, Ondrej Krystynik ${ }^{1}$, Dominika Goldmannova ${ }^{1}$, \\ Lubica Cibickova ${ }^{1}$, Jan Schovanek ${ }^{1}$ \\ ${ }^{1}$ University Hospital and Palacky University, Faculty of Medicine and Dentistry, Third Department of Internal Medicine - Nephrology, Rheumatology \\ and Endocrinology, Olomouc, Czech Republic \\ ${ }^{2}$ University Hospital, Department of Clinical Biochemistry, Olomouc, Czech Republic
}

\begin{abstract}
Backgrounds: Adiponectin, adipocyte-fatty acid binding protein (A-FABP), and Wnt1 inducible signaling pathway protein-1 (WISP-1) are adipokines closely associated with insulin resistance. The aim of the study was to compare their levels in women with gestational diabetes (GDM), type 2 diabetes mellitus (T2DM) and healthy controls and determine their relation to metabolic parameters.

Methods: Women with GDM, T2DM and healthy women were included in this cross-sectional study. In addition to adipokines, anthropometric, lipid parameters, markers of insulin resistance and glucose control were assessed in all participants.

Results: Compared to healthy controls $(n=35)$ significantly lower levels of adiponectin were detected in women with GDM ( $n=50)$, whereas in women with T2DM $(n=50)$ higher levels of A-FABP and WISP-1 and lower levels of adiponectin were found. Women with T2DM had also lower levels of adiponectin and higher levels of A-FABP compared to women with GDM. A-FABP and adiponectin were independently associated with levels of triglycerides, HDL-cholesterol and C-peptide insulin resistance index. WISP-1 correlated only with waist circumference.

Conclusions: Adverse adipokines production reflecting dysfunctional fat tissue is less presented in women with GDM than in women with T2DM, but more expressed compared to healthy women.
\end{abstract}

Keywords: Adipocyte-fatty acid binding protein; Adiponectin; Gestational diabetes; Insulin resistance; Type 2 diabetes; Wnt1 inducible signaling pathway protein-1

\section{Highlights:}

- Dysfunctional fat tissue plays important role in development of insulin resistance.

- Adverse adipokines production is less expressed in GDM than T2DM.

- Low adiponectin could participate in pregnancy complications due to lipid profile.

- High A-FABP and WISP-1 levels correlate with high cardiovascular risk in women with T2DM.

\section{Introduction}

Gestational diabetes mellitus (GDM) is defined as glucose intolerance developing in the second and third trimester of pregnancy in women with no history of diabetes prior to gestation. Pathophysiology of GDM shares some common features with type 2 diabetes mellitus (T2DM) combining relatively insufficient insulin secretion with increased peripheral insulin resistance. Adipose tissue dysfunction is a well-described cause of increased insulin resistance in individuals with T2DM. Emerging evidence of altered adipokines expression in patients with gestational diabetes suggests an important involvement of dysfunctional adipose tissue in its etiopathogenesis (Šimják et al., 2018). Adiponectin and adipocyte-fatty acid binding protein (A-FABP) belong to abundantly expressed adipokines par- ticularly related to insulin resistance (Furuhashi, 2019; Yanai and Yoshida, 2019). Recently, association between relatively novel adipokine - Wnt1 inducible signaling pathway protein-1 (WISP-1) and insulin resistance has also been studied (Yaribeygi et al., 2019). Adiponectin and A-FABP were selected as well-known adipokines with proven relation to insulin resistance and cardiovascular diseases (Novotny et al., 2014; Xu and Vanhoutte, 2012; Yanai and Yoshida, 2019). There is an increasing evidence pointing to WISP-1 as a promising regulator of insulin sensitivity and chronic inflammation (Hörbelt et al., 2018; Murahovschi et al., 2015).

The healthy adipose tissue is a primary source of adiponectin. It is one of the most abundant peptide hormones derived from adipose tissue. This protein plays a major role in glucose and lipid metabolism and prevents development of cardiovascular changes due to its anti-oxidative, anti-inflammatory, en-

\footnotetext{
* Author for correspondence: David Karasek, University Hospital Olomouc, Third Department of Internal Medicine - Nephrology, Rheumatology and Endocrinology, I. P. Pavlova 6, 77900 Olomouc, Czech Republic; e-mail: david.karasek@fnol.cz http://doi.org/10.32725/jab.2020.007

Submitted: 2019-07-29 • Accepted: 2020-03-30 • Prepublished online: 2020-05-18

J Appl Biomed 18/2-3: 54-60 • EISSN 1214-0287 • ISSN 1214-021X

(c) 2020 The Authors. Published by University of South Bohemia in České Budějovice, Faculty of Health and Social Sciences.

This is an open access article under the CC BY-NC-ND license.
} 
dothelium-protective and anti-apoptotic effects (Xu and Vanhoutte, 2012). Adiponectin production is connected with an increase in serum HDL-cholesterol (HDL-C) and it lowers serum triglyceride (TG) levels through the enhanced catabolism of TG-rich lipoproteins (Christou and Kiortsis, 2013). Beneficial effects of adiponectin on glucose metabolism comprise: reduction of inflammation and oxidative stress and improvement of insulin resistance; protective effect on pancreatic $\beta$-cells; increase of glucose utilization and fatty acid oxidation in skeletal muscles; reduction of hepatic glucose production; and increase of insulin-stimulated glucose uptake by adipocytes (Yanai and Yoshida, 2019). Hypoadiponectinemia has been detected in numerous disorders accompanying insulin resistance such as obesity, T2DM, hypertension, mixed dyslipidemia, metabolic syndrome, non-alcoholic steatosis, polycystic ovarian syndrome, etc. (Novotny et al., 2014; Spurná et al., 2018; Xu and Vanhoutte, 2012; Yanai and Yoshida, 2019).

A-FABP is formed mainly in adipocytes and macrophages. Its main function is binding of free fatty acids (Xu and Vanhoutte, 2012). A-FABP appears to reduce contractility of cardiomyocytes, promotes chronic inflammation and formation of vulnerable atherosclerotic plaques (Furuhashi et al., 2015; $\mathrm{Xu}$ and Vanhoutte, 2012). It is involved in the regulation of inflammatory and metabolic processes in target cells. The presence of A-FABP may be beneficial for storing energy in adipocytes, for acting on an immune response in macrophages, and for trafficking of fatty acids in capillary endothelial cells (Furuhashi, 2019). Additionally, secreted A-FABP in association with lipolysis during fasting may regulate hepatic glucose production (Furuhashi, 2019; Furuhashi et al., 2015). It has been reported that increased circulating A-FABP levels are associated with obesity, insulin resistance, dyslipidemia, type 2 diabetes, metabolic syndrome, hypertension, cardiac dysfunction, and atherosclerosis (Furuhashi, 2019; Furuhashi et al., 2015; Novotny et al., 2014; Spurná et al., 2018).

WISP-1 (also known as CCN4) is a member of the secreted extracellular matrix-associated proteins of the CCN family and a target gene of the Wingless-type (WNT) signaling pathway. Growing evidence links the WNT signaling pathway to the regulation of adipogenesis and low-grade inflammation in obesity. WISP1 expression in visceral and subcutaneous fat tissue was associated with markers of insulin resistance and inflammation in glucose-tolerant subjects (Murahovschi et al., 2015). Circulating WISP1 levels were increased in obese persons and are directly related to adiposity, independent of glycemic status or insulin resistance (Barchetta et al., 2017). Adverse effects of WISP-1 on glucose metabolism seems to be mediated by its direct inhibition of phosphorylation of insulin receptor in liver and muscle cells resulting in an increase of liver glucose production and a decrease of muscle glycogen synthesis (Hörbelt et al., 2018).

Based on these facts we could hypothesize, that circulating levels of mentioned adipokines should differ in women with GDM compared to healthy controls and may be similar to patients with T2DM. The aim of this study was to compare adipokines plasma levels in women with GDM and in women with T2DM to healthy controls. Association between adipokines and metabolic profile of the participants were also investigated.

\section{Material and methods}

\section{Study design, inclusion and exclusion criteria}

The study was undertaken as a cross-sectional study with GDM, T2DM patients and healthy controls in accordance with the principles of the Declaration of Helsinki as revised in 2008. It was reviewed and approved by Ethics Committee of Medical Faculty and University Hospital Olomouc and informed consent was obtained from all participants. The diagnosis of GDM was based on any one of the following values at any time during pregnancy: fasting plasma glucose $=5.1-6.9 \mathrm{mmol} / \mathrm{l}$; $1 \mathrm{~h}$ post $75 \mathrm{~g}$ oral glucose load $\geq 10.0 \mathrm{mmol} / \mathrm{l} ; 2 \mathrm{~h}$ post $75 \mathrm{~g}$ oral glucose load 8.5-11.0 mmol/1; according to criteria of the International Association of Diabetic Pregnancy Study Group (IADPSG) guidelines (Metzger et al., 2010). T2DM was defined as fasting plasma glucose $\geq 7 \mathrm{mmol} / 1$ or using of peroral antidiabetic drugs (PADs) and/or insulin. Exclusion criteria were as follows: type 1 diabetes, secondary or genetic type of diabetes, acute infection, or trauma. Controls constituted healthy women without personal history of glucose intolerance or diabetes (including GDM, or history of high birth weight baby ( $\geq 4.5 \mathrm{~kg}$ ) delivery). Participants were asked about their medical history and smoking. Body mass index (BMI), waist circumference, systolic and diastolic blood pressure (SBP and DBP), and laboratory tests were also performed.

\section{Laboratory analyses}

Venous blood samples were drawn in the morning after a $12 \mathrm{~h}$ fast. Routine serum biochemical parameters (lipids, glucose, glycated hemoglobin, C-peptide) were analyzed on Cobas 8000 (Roche, Mannheim, Germany) on the day of blood collection. Concentrations of adipokines were measured in the sample aliquots stored at $-80^{\circ} \mathrm{C}$, no longer than 6 months.

Total cholesterol (TC), TG and HDL-C were determined enzymatically on Cobas 8000 system. Low density lipoprotein cholesterol (LDL-C) was calculated using Friedewald formula (LDL-C = TC - TG*0.4537 - HDL-C for TG < $4.5 \mathrm{mmol} / \mathrm{l})$. Non-HDL-cholesterol was calculated as follows: non-HDL-C $=\mathrm{TC}-\mathrm{HDL}-\mathrm{C}$. Glucose was determined using hexokinase method (Roche, Basel, Switzerland). Glycated hemoglobin levels (HbA1C) were measured by ion exchange chromatography using Arkray Adams HA-8180V analyzer (Arkray Corporation, Kyoto, Japan). C-peptide was determined by the commercially available kit (Immunotech, Marseille, France) using specific antibodies by the IRMA methods. Based on glucose and C-peptide levels so called C-peptide insulin resistance index (CP-IRI = 20/fasting C-peptide ${ }^{*}$ fasting plasma glucose) was calculated (Ohkura et al., 2013). This index should be more effective than HOMA-IR (Homeostasis model assessment of insulin resistance) in patients with mild insulin resistance. Moreover, CP-IRI calculation is not influenced by artificial insulin levels due to insulin administration. CP-IRI is also minimally affected by hepatic insulin clearance (Okura et al., 2018). Moreover, C-peptide-based index seem to be more related to incident T2DM than insulin-based index (Kim et al., 2016).

Adiponectin was determined by ELISA immunochemical kit: Human Adiponectin ELISA (Biovendor Laboratory Medicine Inc., Brno, Czech Republic), according to the manufacturer's instructions. The antibodies used in this kit are specific for human adiponectin. Assay sensitivity is $26 \mathrm{ng} / \mathrm{ml}$. Adipocyte fatty acid binding protein was assessed by immunochemical test: Human Adipocyte FABP4 ELISA kit (Biovendor Laboratory Medicine Inc., Brno, Czech Republic), according to the manufacturer's instructions. The antibodies used in this ELISA are specific for human AFABP. Assay sensitivity is $0.08 \mathrm{ng} / \mathrm{ml}$. WISP-1 was measured by immunochemical kit: Human WISP1 ELISA kit (Thermo Fisher Scientific, Massachusetts, USA), according to the manufacturer's instructions. The antibodies used in this ELISA are specific for human WISP-1. Assay sensitivity is $0.05 \mathrm{ng} / \mathrm{ml}$. The inter- and intra- assay coefficients of variation were below $12 \%$. 


\section{Statistical analyses}

All values were expressed as median and interquartile range (IQR). Differences in variables between the groups were analyzed with ANOVA after the adjustment for age. Spearman correlation analyses tested correlations between parameters in all groups. Multivariate regression analyses were used for testing for an independent association between dependent and independent variables (non-normally distributed variables were logarithmically transformed before analyses). Probability values of $p<0.05$ were considered as statistically significant.

\section{Results}

Fifty women with GDM (age = 33.7 (20.9-36.4) years), 50 non-pregnant women with T2DM (age $=47.3(34.2-53.4)$ years) and 35 non-pregnant healthy controls (age $=40.7$ (31.4-45.8) years) met eligible criteria for this study. Examination of women with GDM was performed on average in the 28.5th (25.4th-29.8th) week of gravidity. Only 3 of them (6\%) were treated with insulin, none of them was treated with metformin. Average duration of diabetes in T2DM group was 5.9 (4.7-8.9) years. Insulin was administered in $44 \%$ of them, $92 \%$ were treated with PADs. The most commonly used PADs were: metformin (92\%), then incretins (56\%, glucagon-like peptide 1 receptor agonists - 8\%, or dipeptidyl peptidase- 4 inhibitors $-48 \%$ ), gliflozins (18\%), and sulfonylurea's (14\%). Seventy-six percent of women with T2DM were treated by hypolipidemic drugs and $82 \%$ of them were treated by anti-hypertensive drugs. Ten percent of T2DM women (5/50) suffered from coronary heart disease, $8 \%$ (4/50) suffered from periphery artery disease and $8 \%(4 / 50)$ of them reported a his- tory of stroke. Prevalence of microvascular complications was $26 \%$ (13/50) for diabetic kidney disease, 40\% (20/50) for diabetic neuropathy and 24\% (12/50) for retinopathy.

Table 1 shows basic clinical and laboratory characteristics of participants. Compared to healthy controls, women with GDM had significantly higher BMI, waist, levels of total cholesterol, LDL-C, non-HDL-C, TG, C-peptide, and CP-IRI. Women with T2DM had significantly higher BMI, waist, SBP, levels of TG, fasting glucose, HbA1C, C-peptide, CP-IRI, and lower levels of HDL-C. In comparison with GDM group, women with T2DM had significantly higher waist, SBP, DBP, levels of fasting glucose, HbA1C, CP-IRI, and lower levels of total cholesterol, LDL-C, HDL-C, and non-HDL-C.

\section{Adipokines in individual groups, their relation to metabolic parameters}

Only levels of adiponectin were significantly decreased in women with GDM compared to healthy controls. There were no significant differences between A-FABP or WISP-1 levels. Women with T2DM had significantly lower levels of adiponectin and higher levels of both A-FABP and WISP-1 compared to healthy controls. Compared to GDM patients, T2DM subjects had significantly lower levels of adiponectin and higher levels of A-FABP - see Table 1 and Fig. 1.

Adiponectin significantly $(p<0.05)$ correlated positively with HDL-C ( $\rho=0.68)$ and CP-IRI $(\rho=0.61)$; negatively with $\operatorname{BMI}(\rho=-0.40)$, waist $(\rho=-0.51)$, TG $(\rho=-0.52)$, fasting glucose $(\rho=-0.48), \operatorname{HbA1C}(\rho=-0.47), C$-peptide $(\rho=-0.51)$ and age $(\rho=-0.19)$. A-FABP negatively correlated with HDL-C $(\rho=-0.57)$ and CP-IRI $(\rho=-0.65)$; positively with BMI $(\rho=$ $0.60)$, waist $(\rho=0.65)$, SBP $(\rho=0.31)$, DBP $(\rho=0.17)$, smoking ( $\rho=0.17)$, TG $(\rho=0.17)$, fasting glucose $(\rho=0.60)$, HbA1C

Table 1. Basic clinical and laboratory characteristics in individual groups

\begin{tabular}{lccc}
\hline & $\begin{array}{c}\text { Healthy controls } \\
(n=35)\end{array}$ & $\begin{array}{c}\text { GDM group } \\
(n=50)\end{array}$ & $\begin{array}{c}\text { T2DM group } \\
(n=50)\end{array}$ \\
\hline BMI $\left(\mathrm{kg} / \mathrm{m}^{2}\right)$ & $22.2(20.2-25.9)$ & $28.8(24.3-32.9)^{\mathrm{a}}$ & $31.6(29.6-36.6)^{\mathrm{b}}$ \\
Waist $(\mathrm{cm})$ & $76.0(70.1-86.2)$ & $100.0(93.5-107.4)^{\mathrm{a}}$ & $108.5(104.5-119.0)^{\mathrm{b}, \mathrm{c}}$ \\
SBP $(\mathrm{mm} \mathrm{Hg})$ & $120.9(110.1-135.5)$ & $122.6(111.5-131.7)$ & $133.1(125.0-140.9)^{\mathrm{b}, \mathrm{c}}$ \\
DBP $(\mathrm{mm} \mathrm{Hg})$ & $78.2(75.4-84.6)$ & $75.5(69.8-81.4)$ & $80.2(77.0-87.6)^{\mathrm{c}}$ \\
TC $(\mathrm{mmol} / \mathrm{l})$ & $4.9(4.7-5.6)$ & $6.3(5.5-7.1)^{\mathrm{a}}$ & $4.3(3.9-5.1)^{\mathrm{c}}$ \\
LDL-C $(\mathrm{mmol} / \mathrm{l})$ & $2.6(2.2-3.3)$ & $3.2(2.7-4.0)^{\mathrm{a}}$ & $2.3(1.8-2.9)^{\mathrm{c}}$ \\
HDL-C $(\mathrm{mmol} / \mathrm{l})$ & $1.9(1.4-2.3)$ & $1.9(1.5-2.3)$ & $1.1(0.9-1.4)^{\mathrm{b}, \mathrm{c}}$ \\
non-HDL-C $(\mathrm{mmol} / \mathrm{l})$ & $3.1(2.5-4.0)$ & $4.2(3.6-5.1)^{\mathrm{a}}$ & $3.2(2.7-3.9)^{\mathrm{c}}$ \\
TG $(\mathrm{mmol} / \mathrm{l})$ & $1.0(0.7-1.3)$ & $2.3(1.8-3.0)^{\mathrm{a}}$ & $2.1(1.4-2.4)^{\mathrm{b}}$ \\
FPG $(\mathrm{mmol} /)$ & $4.6(4.3-5.0)$ & $4.7(4.3-5.0)$ & $8.7(6.8-11.4)^{\mathrm{b}, c}$ \\
HbA1C $(\mathrm{mmol} / \mathrm{mol})$ & $32.0(29.6-34.4)$ & $32.0(30.5-34.2)$ & $66.5(47.9-83.5)^{\mathrm{b}, c}$ \\
C-peptide $(\mathrm{pmol} / \mathrm{l})$ & $425.5(355.7-576.0)$ & $613.1(487.5-906.0)^{\mathrm{a}}$ & $846.0(608.0-1083.6)^{\mathrm{b}}$ \\
CP-IRI & $10.1(7.0-13.4)$ & $6.9(4.9-9.3)^{\mathrm{a}}$ & $2.9(1.9-3.7)^{\mathrm{b}, c}$ \\
Adiponectin $(\mu \mathrm{gg} / \mathrm{ml})$ & $11.6(9.4-14.9)$ & $9.1(7.7-10.3)^{\mathrm{a}}$ & $5.8(4.0-8.2)^{\mathrm{b}, c}$ \\
A-FABP $(\mathrm{ng} / \mathrm{ml})$ & $25.5(21.1-31.9)$ & $21.8(13.3-31.2)$ & $59.7(40.9-70.1)^{\mathrm{b}, c}$ \\
WISP-1 $(\mathrm{pg} / \mathrm{ml})$ & $35.6(29.0-61.1)$ & $39.0(28.8-49.4)$ & $54.4(32.8-82.9)^{\mathrm{b}}$ \\
\hline
\end{tabular}

GDM, gestational diabetes mellitus; T2DM, type 2 diabetes mellitus; BMI, body mass index; SBP, systolic blood pressure; DBP, diastolic blood pressure; TC, total cholesterol; LDL-C, LDL-cholesterol; HDL-C, HDL-cholesterol; non-HDL-C, non-HDL-cholesterol; TG, triglycerides; FPG, fasting plasma glucose; HbA1C, glycated hemoglobin A1C; CP-IRI, 20 / FPG (mmol/l) * C-peptide (nmol/l); A-FABP, adipocyte-fatty acid binding protein; WISP-1, Wnt1 inducible signaling pathway protein-1.

Values are expressed as median ( 25 and 75 percentile). Significant differences $(p<0.05)$ according to ANOVA (after adjustment for age): ${ }^{\text {a GDM vs }}$ controls; ${ }^{\mathrm{b}}$ T2DM vs controls; ${ }^{\mathrm{c}} \mathrm{T}$ T2DM vs GDM. 


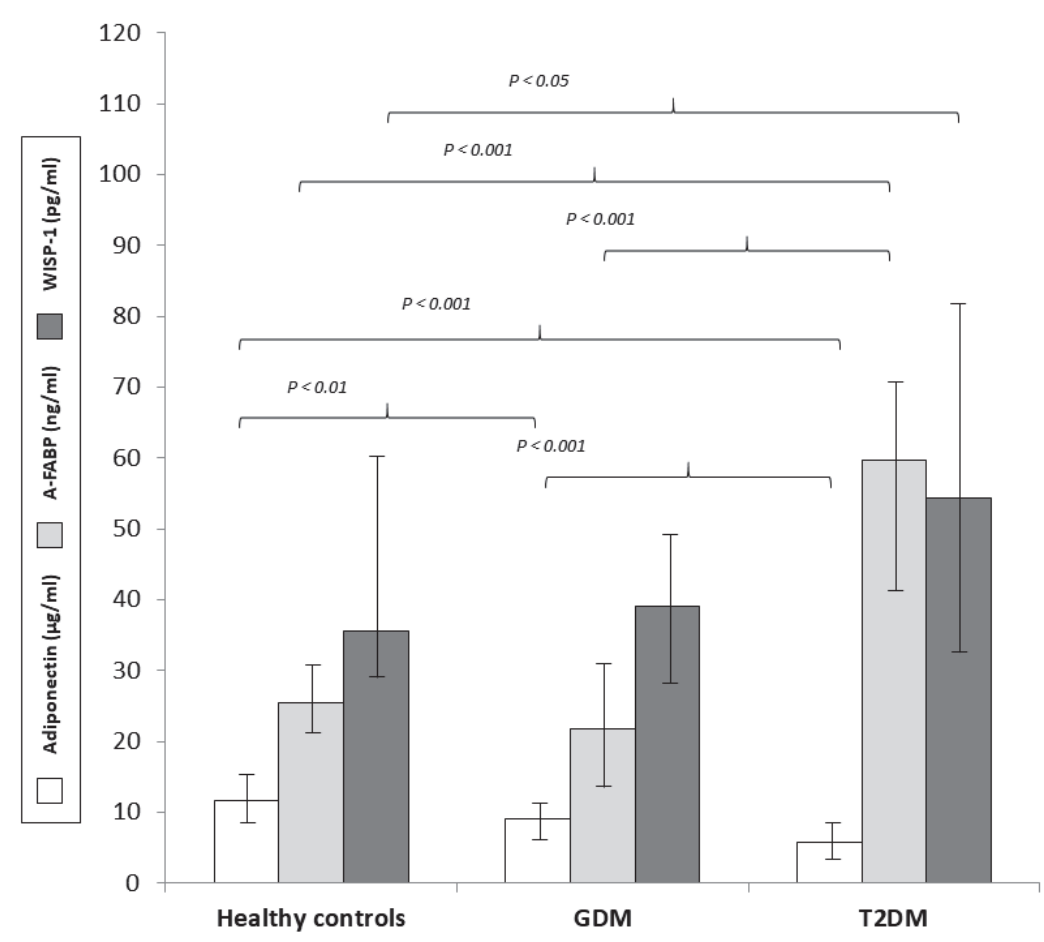

Fig. 1. Levels of adipokines in different group (medians - bar plots, 25 and 75 percentiles - error bars).

GDM, gestational diabetes mellitus; T2DM, type 2 diabetes mellitus; A-FABP, adipocyte-fatty acid binding protein; WISP-1, Wnt1 inducible signaling pathway protein-1.

( $\rho=0.68)$, C-peptide $(\rho=0.44)$, and age $(\rho=0.49)$. WISP-1 correlated only with waist $(\rho=0.19)$. There was also a negative correlation between adiponectin and A-FABP $(\rho=-0.39)$, WISP-1 $(\rho=-0.19)$, respectively; and a positive correlation between A-FABP and WISP-1 $(\rho=0.33)$.

Table 2 shows multivariate logistic regression analysis of independent factors affecting adiponectin and/or A-FABP as dependent variables. Both these adipokines were independently associated with levels of TG, HDL-C and CP-IRI.

\section{Discussion}

Women with GDM had only significantly lower levels of adiponectin, while women with T2DM had above that higher levels of A-FABP and WISP-1 compared to healthy controls. Moreover, their A-FABP levels were significantly higher and adiponectin levels significantly lower than levels of these adipokines in women with GDM. These results suggested more

Table 2. Multivariate logistic regression analysis of independent factors affecting adiponectin and/or adipocyte-fatty acid binding protein as dependent variables

\begin{tabular}{|c|c|c|c|c|c|c|}
\hline & \multicolumn{3}{|c|}{ Adiponectin } & \multicolumn{3}{|c|}{ Adipocyte-fatty acid binding protein } \\
\hline & $B$ & Std. Error & Sig. & $B$ & Std. Error & Sig. \\
\hline age & 0.138 & 0.215 & 0.524 & 0.538 & 0.213 & 0.014 \\
\hline BMI & 0.574 & 0.459 & 0.214 & 0.671 & 0.437 & 0.128 \\
\hline waist & 0.067 & 0.455 & 0.883 & 0.666 & 0.553 & 0.232 \\
\hline TG & -0.274 & 0.113 & 0.017 & -0.353 & 0.112 & 0.002 \\
\hline HDL-C & 0.491 & 0.179 & 0.007 & -0.456 & 0.164 & 0.007 \\
\hline FPG & 0.382 & 0.298 & 0.204 & 0.073 & 0.289 & 0.801 \\
\hline $\mathrm{HbA1C}$ & -0.514 & 0.286 & 0.076 & 0.019 & 0.275 & 0.946 \\
\hline CP-IRI & 0.292 & 0.110 & 0.009 & -0.264 & 0.112 & 0.021 \\
\hline SBP & - & - & - & 0.006 & 0.499 & 0.991 \\
\hline DBP & - & - & - & -0.735 & 0.475 & 0.125 \\
\hline smoking & - & - & - & -0.059 & 0.063 & 0.354 \\
\hline
\end{tabular}

BMI, body mass index; TG, triglycerides; HDL-C, HDL-cholesterol; FPG, fasting plasma glucose; HbA1C, glycated hemoglobin A1C; SBP, systolic blood pressure; DBP, diastolic blood pressure; CP-IRI, 20/FPG (mmol/l); ${ }^{*}$ C-peptide (nmol/l). 
considerable contribution of dysfunctional fat tissue to insulin resistance in women with T2DM than in women with GDM.

Compared to healthy women, women with GDM were more insulin resistant and showed signs of mixed dyslipidemia. Studies mostly suggest that women with GDM have increased levels of TG, lower levels of HDL-C and higher levels of nonHDL-C, while levels of LDL-C and total cholesterol are generally inconsistently increased, not related to insulin resistance (Ryckman et al., 2015). It is unclear as to whether disturbances in lipid metabolism occur exclusively later in pregnancy after the manifestation of GDM or if they exist before the manifestation of insulin resistance, however mixed dyslipidemia, particularly hypertriglyceridemia, is thought to be one of the key drivers of fetal macrosomia (Herrera and Ortega-Senovilla, 2010). Macrosomia of newborns is also connected with decreased adiponectin, and maternal adiponectin levels are inversely associated with neonatal birth body weight (Šimják et al., 2018). Low adiponectin levels in women with GDM were previously found in numerous studies and hypoadiponectinemia at least could be considered as a predictive marker of GDM development in early pregnancy (Bao et al., 2015; Iliodromiti et al., 2016). Hypoadiponectinemia during pregnancy predicts postpartum insulin resistance (Retnakaran et al., 2010), and future unfavorable cardiovascular risk profile (especially adverse lipid profile with high TG/HDL-C ratio) (Lekva et al., 2017). Despite increased insulin resistance, no significant alterations in adiponectin levels were observed during physiological pregnancy and adiponectin levels were similar at 1st, 2nd and 3rd trimester, and significantly decreased in postpartum period (Mazaki-Tovi et al., 2007). Thus, low adiponectin levels (together with their independent association with TG, HDL-C, and C-peptide insulin resistance index documented in this study) support significance of dysfunctional fat tissue in onset of GDM and its complications.

On the contrary, women with GDM did not differ in levels of A-FABP and of WISP-1 compared to healthy controls in this study. Several authors previously found increased A-FABP in women with GDM (Kralisch et al., 2009; Ortega-Senovilla et al., 2011). In the present study, we used the IADPSG criteria, which distinguished it from these previous studies. Using the IADPSG criteria resulted in a rise of GDM prevalence, because women with only slight hyperglycemia have been also identified as subjects with GDM. These women could be less insulin resistant compared to previously diagnosed GDM patients. However, two Chinese cohorts of women with GDM based on the IADPSG criteria showed increased A-FABP levels too (Li et al., 2015; Zhang et al., 2016). The genetic background of our study participants coming from Caucasian population, which is different from both those studies, could explain the differences in study results. Recently, Sahin Ersoy et al. (2017) observed also significantly higher circulating WISP-1 in women with GDM group, which were independently predicted by BMI and HOMA-IR. Insulin resistance in women with GDM of this study was not so expressed like in women of that mentioned study. Their WISP-1 levels were only non-significantly elevated and correlated with waist circumference, but not with CP-IRI. These results are supported by findings of Barchetta et al. (2017), who detected an increase of WISP-1 levels in obese persons, which were directly related to adiposity, independent of glycemic status or insulin resistance.

Significantly higher A-FABP and WISP-1 levels compared to healthy women were detected only in women with T2DM. They had also higher A-FABP levels compared to women with GDM. A-FABP correlated with markers of abdominal obesity, dyslipidemia, insulin resistance, arterial hypertension, smok- ing and age. Association between A-FABP and main cardiovascular risk factors, together with its direct atherogenic and pro-inflammatory effects, ranks it to adipokines with the most adverse cardiovascular impact (Furuhashi et al., 2015; Xu and Vanhoutte, 2012). A-FABP levels were independently associated with coronary atherosclerotic burden (Miyoshi et al., 2010) and especially serum A-FABP/adiponectin ratio may be a useful indicator for risk of coronary artery disease (CAD) (Jin et al., 2010). WISP-1 levels may be also related to cardiovascular damage. WISP-1 was increased in patients with myocardial infarction (Wright et al., 2018) and CAD, its levels correlated with severity of CAD (Gu et al., 2019). Reduced WISP-1 levels in mouse carotid arteries from WISP-1 ${ }^{-/-}$mice, significantly suppressed intimal thickening in response to carotid artery ligation. In contrast, via an adenovirus encoding WISP-1 significantly increased intimal thickening compared with mice receiving control virus (Williams et al., 2016). Therefore, high levels of A-FABP and of WISP-1, and low adiponectin levels reflect the high cardiovascular risk of T2DM women, not only due to diabetes, visceral obesity and insulin resistance, but because of the accumulation of other risk factors such as hypertension and dyslipidemia. Although substantial part of T2DM women were treated by hypolipidemic and anti-hypertensive drugs, they still showed signs of atherogenic dyslipidemia and higher SBP compared to healthy controls.

The first limitation of this study is its cross-sectional design. Samples were obtained only during the second trimester of pregnancy, so we do not know adipokines levels at the first and third trimesters. For example, there was a significant increase in A-FABP from the second to third trimester in women with GDM (Zhang et al., 2016). The second, GDM is heterogenous disease with different contribution of insulin resistance and/or insulin secretion impairment (Powe et al., 2016). Therefore, different phenotypes of GDM based on those pathophysiological mechanisms could be associated with different adipokines production. This may be a target for prospective study investigating the longitudinal changes of these molecules in different GDM phenotypes during pregnancy. Strength of this study is the enrolling of not only the healthy controls, but also women with T2DM, that could help to identify similarities and dissimilarities between GDM and T2DM pathogeneses.

\section{Conclusions}

Significantly lower adiponectin levels in GDM women reflect contribution of impaired insulin sensitivity, which via adverse lipid profile could participate on short- and long-term adverse health outcomes for both women and their children. High levels of A-FABP and of WISP-1 together with low adiponectin levels in T2DM women verify their high cardiovascular risk and point on more considerable contribution of dysfunctional fat tissue to insulin resistance in pathogenesis of T2DM.

\section{Conflict of interests}

The authors have no conflict of interests to declare.

\section{Acknowledgements and funding}

Authors would like to thank RNDr. Milena Krskova for assistance with statistical analysis. Financial support of this study was provided by Czech health research council (AZV NV1801-00139) and University Hospital Olomouc - MH CZ DRO (FNOl, 00098892). Funding sources were not involved in the study design, in the collection, analysis and interpretation of data, in the writing of the report, and in the decision to submit the article for publication. 


\section{References}

Bao W, Baecker A, Song Y, Kiely M, Liu S, Zhang C (2015). Adipokine levels during the first or early second trimester of pregnancy and subsequent risk of gestational diabetes mellitus: A systematic review. Metabolism 64: 756-764. DOI: 10.1016/j. metabol.2015.01.013.

Barchetta I, Cimini FA, Capoccia D, De Gioannis R, Porzia A, Mainiero F, et al. (2017). WISP1 Is a Marker of Systemic and Adipose Tissue Inflammation in Dysmetabolic Subjects With or Without Type 2 Diabetes. J Endocr Soc 1: 660-670. DOI: 10.1210/ js.2017-00108.

Christou GA, Kiortsis DN (2013). Adiponectin and lipoprotein metabolism. Obes Rev 14: 939-949. DOI: 10.1111/obr.12064.

Declaration of Helsinki: Ethical Principles for Medical Research Involving Human Subjects (2008). The World Medical Association, Inc. [online] [cit. 2020-05-07]. Available at: https://www.wma.net/ wp-content/uploads/2016/11/DoH-Oct2008.pdf

Furuhashi M (2019). Fatty Acid-Binding Protein 4 in Cardiovascular and Metabolic Diseases. J Atheroscler Thromb 26: 216-232. DOI: $10.5551 /$ jat. 48710.

Furuhashi M, Saitoh S, Shimamoto K, Miura T (2015). Fatty AcidBinding Protein 4 (FABP4): Pathophysiological Insights and Potent Clinical Biomarker of Metabolic and Cardiovascular Diseases. Clin Med Insights Cardiol 8(Suppl. 3): 23-33. DOI: 10.4137/CMC. S17067.

Gu L, Chen N, Li Z, Zhang F, Wang X (2019). Relationship of serum Wnt1-inducible signaling pathway protein 1 levels with coronary artery disease and its severity. Coron Artery Dis 30(7): 542-546. DOI: 10.1097/MCA.0000000000000745.

Herrera E, Ortega-Senovilla H (2010). Disturbances in lipid metabolism in diabetic pregnancy - Are these the cause of the problem? Best Pract Res Clin Endocrinol Metab 24: 515-525. DOI: 10.1016/j.beem.2010.05.006.

Hörbelt T, Tacke C, Markova M, Herzfeld de Wiza D, Van de Velde F, Bekaert M, et al. (2018). The novel adipokine WISP1 associates with insulin resistance and impairs insulin action in human myotubes and mouse hepatocytes. Diabetologia 61: 2054-2065. DOI: 10.1007/s00125-018-4636-9.

Iliodromiti S, Sassarini J, Kelsey TW, Lindsay RS, Sattar N, Nelson SM (2016). Accuracy of circulating adiponectin for predicting gestational diabetes: a systematic review and metaanalysis. Diabetologia 59: 692-699. DOI: 10.1007/s00125-0153855-6.

Jin J, Peng DQ, Yuan SG, Zhao SP, Ning XH, Wang SH, Li SL (2010). Serum adipocyte fatty acid binding proteins and adiponectin in patients with coronary artery disease: the significance of A-FABP/adiponectin ratio. Clin Chim Acta 411: 1761-1765. DOI: 10.1016/j.cca.2010.07.031.

Kim JD, Kang SJ, Lee MK, Park SE, Rhee EJ, Park CY, et al. (2016). C-Peptide-Based Index Is More Related to Incident Type 2 Diabetes in Non-Diabetic Subjects than Insulin-Based Index. Endocrinol Metab (Seoul) 31: 320-327. DOI: 10.3803/ EnM.2016.31.2.320.

Kralisch S, Stepan H, Kratzsch J, Verlohren M, Verlohren HJ, Drynda K, et al. (2009). Serum levels of adipocyte fatty acid binding protein are increased in gestational diabetes mellitus. Eur J Endocrinol 160: 33-38. DOI: 10.1530/EJE-08-0540.

Lekva T, Michelsen AE, Aukrust P, Henriksen T, Bollerslev J, Ueland T (2017). Leptin and adiponectin as predictors of cardiovascular risk after gestational diabetes mellitus. Cardiovasc Diabetol 16: 5. DOI: 10.1186/s12933-016-0492-4.

Li YY, Xiao R, Li CP, Huangfu J, Mao JF (2015). Increased plasma levels of FABP4 and PTEN is associated with more severe insulin resistance in women with gestational diabetes mellitus. Med Sci Monit 21: 426-431. DOI: 10.12659/MSM.892431.

Mazaki-Tovi S, Kanety H, Pariente C, Hemi R, Wiser A, Schiff E, Sivan E (2007). Maternal serum adiponectin levels during human pregnancy. J Perinatol 27: 77-81. DOI: 10.1038/sj.jp.7211639.

Metzger BE, Gabbe SG, Persson B, Buchanan TA, Lowe LP, Dyer AR, et al. (2010). International association of diabetes and pregnancy study groups recommendations on the diagnosis and classification of hyperglycemia in pregnancy. Diabetes Care 33(3): 676-682. DOI: $10.2337 / \mathrm{dc} 09-1848$.

Miyoshi T, Onoue G, Hirohata A, Hirohata S, Usui S, Hina K, et al. (2010). Serum adipocyte fatty acid-binding protein is independently associated with coronary atherosclerotic burden measured by intravascular ultrasound. Atherosclerosis 211: 164-169. DOI: 10.1016/j.atherosclerosis.2010.01.032.

Murahovschi V, Pivovarova O, Ilkavets I, Dmitrieva RM, Döcke S, Keyhani-Nejad F, et al. (2015). WISP1 is a novel adipokine linked to inflammation in obesity. Diabetes 64: 856-866. DOI: 10.2337/ db14-0444.

Novotny D, Vaverkova H, Karasek D, Lukes J, Slavik L, Malina P, Orsag J (2014). Evaluation of total adiponectin, adipocyte fatty acid binding protein and fibroblast growth factor 21 levels in individuals with metabolic syndrome. Physiol Res 63: 219-228.

Ohkura T, Shiochi H, Fujioka Y, Sumi K, Yamamoto N, Matsuzawa K, et al. (2013). 20/(fasting C-peptide $\times$ fasting plasma glucose) is a simple and effective index of insulin resistance in patients with type 2 diabetes mellitus: a preliminary report. Cardiovasc Diabetol 12: 21. DOI: $10.1186 / 1475-2840-12-21$

Okura T, Nakamura R, Fujioka Y, Kawamoto-Kitao S, Ito Y, Matsumoto K, et al. (2018). CPR-IR is an insulin resistance index that is minimally affected by hepatic insulin clearance-A preliminary research. PLoS One 13, e0197663. DOI: 10.1371/ journal.pone.0197663.

Ortega-Senovilla H, Schaefer-Graf U, Meitzner K, Abou-Dakn M, Graf K, Kintscher U, Herrera E (2011). Gestational diabetes mellitus causes changes in the concentrations of adipocyte fatty acid-binding protein and other adipocytokines in cord blood. Diabetes Care 34: 2061-2066. DOI: 10.2337/dc11-0715.

Powe CE, Allard C, Battista MC, Doyon M, Bouchard L, Ecker JL, et al. (2016). Heterogeneous Contribution of Insulin Sensitivity and Secretion Defects to Gestational Diabetes Mellitus. Diabetes Care 39: 1052-1055. DOI: 10.2337/dc15-2672.

Retnakaran R, Qi Y, Connelly PW, Sermer M, Hanley AJ, Zinman B (2010). Low adiponectin concentration during pregnancy predicts postpartum insulin resistance, beta cell dysfunction and fasting glycaemia. Diabetologia 53: 268-276. DOI: 10.1007/s00125-009$1600-8$.

Ryckman KK, Spracklen CN, Smith CJ, Robinson JG, Saftlas AF (2015). Maternal lipid levels during pregnancy and gestational diabetes: a systematic review and meta-analysis. BJOG 122: 643-651. DOI: 10.1111/1471-0528.13261.

Sahin Ersoy G, Altun Ensari T, Subas S, Giray B, Simsek EE, Cevik O (2017). WISP1 is a novel adipokine linked to metabolic parameters in gestational diabetes mellitus. J Matern Fetal Neonatal Med 30: 942-946. DOI: 10.1080/14767058.2016.1192118.

Šimják P, Cinkajzlová A, Anderlová K, Pařízek A, Mráz M, Kršek M, Haluzík M (2018). The role of obesity and adipose tissue dysfunction in gestational diabetes mellitus. J Endocrinol 238: R63-R77. DOI: 10.1530/JOE-18-0032.

Spurná J, Karásek D, Kubíčková V, Goldmannová D, Krystyník O, Schovánek J, Zadražil J (2018). Relationship of Selected Adipokines with Markers of Vascular Damage in Patients with Type 2 Diabetes. Metab Syndr Relat Disord 16: 246-253. DOI: 10.1089/met.2017.0179.

Williams H, Mill CA, Monk BA, Hulin-Curtis S, Johnson JL, George SJ (2016). Wnt2 and WISP-1/CCN4 Induce Intimal Thickening via Promotion of Smooth Muscle Cell Migration. Arterioscler Thromb Vasc Biol 36: 1417-1424. DOI: 10.1161/ ATVBAHA.116.307626.

Wright LH, Herr DJ, Brown SS, Kasiganesan H, Menick DR (2018). Angiokine Wisp- 1 is increased in myocardial infarction and regulates cardiac endothelial signaling. JCI Insight 3(4): 95824. DOI: 10.1172/jci.insight.95824.

Xu A, Vanhoutte PM (2012). Adiponectin and adipocyte fatty acid binding protein in the pathogenesis of cardiovascular disease. Am J Physiol Heart Circ Physiol 302: H1231-H1240. DOI: 10.1152/ ajpheart.00765.2011.

Yanai H, Yoshida H (2019). Beneficial Effects of Adiponectin on Glucose and Lipid Metabolism and Atherosclerotic Progression: Mechanisms and Perspectives. Int J Mol Sci 20(5): E1190. DOI: $10.3390 / \mathrm{ijms} 20051190$. 
Yaribeygi H, Atkin SL, Sahebkar A (2019). Wingless-type inducible signaling pathway protein-1 (WISP1) adipokine and glucose homeostasis. J Cell Physiol 234: 16966-16970. DOI: 10.1002/ jcp.28412.
Zhang Y, Zhang HH, Lu JH, Zheng SY, Long T, Li YT, Wu WZ, Wang F (2016). Changes in serum adipocyte fatty acid-binding protein in women with gestational diabetes mellitus and normal pregnant women during mid- and late pregnancy. J Diabetes Investig 7: 797-804. DOI: 10.1111/jdi.12484 